\title{
MONITORING HEALTH BEHAVIOURS AND HEALTH STATUS IN NEW SOUTH WALES: RELEASE OF THE ADULT HEALTH SURVEY 2002
}

\author{
Deborah Baker and Margo Eyeson-Annan \\ Centre for Epidemiology and Research \\ NSW Department of Health
}

In 2002, the NSW Department of Health, in conjunction with the 17 area health services, completed the first year of the New South Wales Continuous Health Survey. This is an ongoing survey of the health of people in NSW that uses computer-assisted telephone interviewing (CATI). The main aims of the Continuous Health Survey are to provide detailed information on the health of the people of NSW, and to support the planning, implementation, and evaluation of health services and programs in NSW. Following the 1997 and 1998 health surveys, this is the third survey that has collected data on the health of adults in NSW. This article announces the release of the report of the New South Wales Adult Health Survey 2002 as a supplementary issue of the NSW Public Health Bulletin, and summarises changes in health behaviour, health status, and satisfaction with health services, which have occurred between 1997 and 2002 .

\section{METHODOLOGY}

The content of the New South Wales Continuous Health Survey was developed by the Health Survey Program in consultation with the Health Survey Program Steering Committee, area health services, other government departments, and a range of experts. The survey content covers the eight priority areas outlined in Healthy People 2005: New Directions for Public Health in New South Wales. Data were collected on a range of health behaviours, health status, use of and satisfaction with health services, social capital, and demographic information. The survey questionnaire was translated into five languages: Arabic, Chinese, Greek, Italian, and Vietnamese.

The target population sampled in the New South Wales Adult Health Survey 2002 was all NSW residents aged 16 years and over living in households with private telephones. Households were sampled using list-assisted random digit dialling. When a household was contacted, one person was randomly-selected for interview. Information was collected on a total of 12,622 adults.

\section{HEALTH BEHAVIOURS}

Unhealthy behaviours contribute significantly to the burden of ill health in NSW. Behaviours that influence health measured in the New South Wales Adult Health Survey 2002 included fruit and vegetable consumption, physical activity, alcohol intake, smoking, and smoking in the home (Table 1).
Just under half of all people in 2002 reported eating the recommended daily fruit intake, while only one in seven people reported consuming the recommended daily intake of vegetables. The proportion of people eating the recommended daily fruit intake (45.3 per cent) and vegetable intake (16.2 per cent) did not change between 1997 and 2002. In 2002, less than a half of the respondents reported using low-fat milk. Since 1997 there has been a reduction in the proportion of people who consume reduced- or low-fat milk (45.7 per cent to 43.4 per cent).

Less than half of all people aged 16 years and over reported undertaking adequate levels of physical activity in 2002. This proportion has not changed since 1997.

In 2002, approximately one-third of the overall adult population reported undertaking risk-drinking behaviours. Encouragingly, since 1997 there has been a notable reduction in the proportion of people who participate in any risk drinking behaviour (42.3 per cent to 34.4 per cent)

In 2002, just over one in five adults reported that they are current smokers, while more than 80 per cent of people reported that their home was smoke-free. Encouragingly, from 1997 to 2002, this represents a reduction in the prevalence of smoking ( 24.0 per cent to 21.4 per cent) and an increase in the proportion of smoke-free households (69.8 per cent to 81.0 per cent).

A number of indicators have been reported for the first time. These include high risk drinking, food security, and vaccinations for influenza and pneumococcal pneumonia in people over 65 years. Trends in these new indicators will continue to be monitored in future surveys. In addition, a comprehensive range of environmental exposures are reported, including exposure to indoor air pollution, wood smoke via wood fires, and benzene via garages attached to home; use of public water supplies, water quality, and exposure to blue green algae through recreational water use; mosquito exposure; and chemical sensitivity.

\section{HEALTH STATUS}

Monitoring the health status of a population helps to detect emerging patterns of illness and disease and provides information to inform policy and planning of health services. The New South Wales Adult Health Survey 2002 collected information on a range of health indicators including: self-rated health status, asthma, diabetes, oral health, overweight and obesity, and psychological distress (Table 2). 


\section{TABLE 1}

\section{TRENDS IN INDICATORS OF HEALTH BEHAVIOURS BY SEX, NSW, 1997-2002}

\begin{tabular}{|c|c|c|c|c|c|c|c|}
\hline \multirow{2}{*}{$\begin{array}{l}\text { Indicator } \\
\text { Alcohol risk drinking }\end{array}$} & \multirow{2}{*}{$\begin{array}{c}\begin{array}{c}\text { Year } \\
\text { Collected }\end{array} \\
1997\end{array}$} & \multirow{2}{*}{$\begin{array}{c}\begin{array}{c}\text { Males } \\
\%\end{array} \\
50.7\end{array}$} & \multirow{2}{*}{$\begin{array}{c}(95 \% \mathrm{Cl}) \\
(49.3-52.2)\end{array}$} & \multicolumn{2}{|c|}{$\begin{array}{l}\text { Females }(95 \% \mathrm{Cl}) \\
\%\end{array}$} & \multicolumn{2}{|c|}{$\begin{array}{l}\text { Persons } \\
\%\end{array}$} \\
\hline & & & & 34.1 & $(32.9-35.4)$ & 42.3 & $(41.3-43.3)$ \\
\hline & 1998 & 50.5 & $(49.0-52.1)$ & 36.2 & $(34.9-37.5)$ & 43.2 & $(42.2-44.2)$ \\
\hline & 2002 & 39.2 & $(37.3-41.1)$ & 29.7 & $(28.1-31.2)$ & 34.4 & $(33.1-35.6)$ \\
\hline High risk drinking in the past 4 weeks & 2002 & 16.7 & $(15.0-18.4)$ & 11.7 & $(10.3-13.1)$ & 14.4 & $(13.3-15.5)$ \\
\hline \multirow[t]{3}{*}{ Pap smear test within the last 2 years } & 1997 & - & - & - & - & - & - \\
\hline & 1998 & - & - & 77.3 & $(75.9-78.7)$ & 77.3 & $(75.9-78.7)$ \\
\hline & 2002 & - & - & 74.6 & $(72.8-76.4)$ & 74.6 & $(72.8-76.4)$ \\
\hline \multirow[t]{3}{*}{ Screening mammogram within the last 2 years } & 1997 & - & - & 73.3 & $(70.9-75.7)$ & 73.3 & $(70.9-75.7)$ \\
\hline & 1998 & - & - & 76.4 & $(74.1-78.7)$ & 76.4 & $(74.1-78.7)$ \\
\hline & 2002 & - & - & 75.2 & $(72.6-77.8)$ & 75.2 & $(72.6-77.8)$ \\
\hline \multirow[t]{3}{*}{ Hysterectomy rate } & 1997 & - & - & 13.3 & $(12.4-14.1)$ & 13.3 & $(12.4-14.1)$ \\
\hline & 1998 & - & - & 13.0 & $(12.2-13.9)$ & 13.0 & $(12.2-13.9)$ \\
\hline & 2002 & - & - & 12.1 & $(11.1-13.1)$ & 12.1 & $(11.1-13.1)$ \\
\hline Use public water as usual source of water & 2002 & - & - & - & - & 81.1 & $(79.5-82.6)$ \\
\hline $\begin{array}{l}\text { Recreational water use limited by blue green algae } \\
\text { in last } 12 \text { months }\end{array}$ & 2002 & - & - & - & - & 6.9 & $(5.9-7.9)$ \\
\hline Gas cooking without ventilation & 2002 & - & - & - & - & 55.6 & $(52.9-58.3)$ \\
\hline Exposure to unflued heating & 2002 & - & - & - & - & 22.6 & $(20.6-24.7)$ \\
\hline $\begin{array}{l}\text { Potential exposure to benzene through } \\
\text { internally-accessed garages }\end{array}$ & 2002 & - & - & - & - & 22.2 & $(20.0-24.3)$ \\
\hline \multirow[t]{3}{*}{ Vaccinated against influenza in the last 12 months } & 1997 & 55.8 & $(52.3-59.2)$ & 58.2 & $(55.3-61.0)$ & 57.1 & $(54.9-59.3)$ \\
\hline & 1998 & 61.9 & $(58.5-65.3)$ & 64.5 & $(61.8-67.2)$ & 63.3 & $(61.2-65.5)$ \\
\hline & 2002 & 75.3 & $(72.4-78.3)$ & 75.7 & $(73.0-78.3)$ & 75.5 & $(73.5-77.5)$ \\
\hline $\begin{array}{l}\text { Vaccinated against pneumococcal disease } \\
\text { in the last } 5 \text { years }\end{array}$ & 2002 & 36.7 & $(33.3-40.1)$ & 41.5 & $(38.5-44.4)$ & 39.4 & $(37.2-41.6)$ \\
\hline \multirow[t]{3}{*}{ Homes with a smoke alarm or detector * } & 1997 & & & & & 58.2 & $(57.2-59.1)$ \\
\hline & 1998 & & & & & 64.0 & $(63.0-65.0)$ \\
\hline & 2002 & & & & & 72.9 & $(71.8-74.0)$ \\
\hline \multirow[t]{3}{*}{ Recommended daily fruit intake } & 1997 & 37.8 & $(36.4-39.2)$ & 51.1 & $(49.8-52.4)$ & 44.5 & $(43.6-45.5)$ \\
\hline & 1998 & 38.0 & $(36.5-39.5)$ & 49.2 & $(47.9-50.5)$ & 43.7 & $(42.7-44.7)$ \\
\hline & 2002 & 40.3 & $(38.4-42.2)$ & 50.1 & $(48.4-51.7)$ & 45.3 & $(44.0-46.5)$ \\
\hline \multirow[t]{3}{*}{ Recommended vegetable intake } & 1997 & 10.8 & $(10.0-11.7)$ & 21.7 & $(20.6-22.7)$ & 16.3 & $(15.6-17.0)$ \\
\hline & 1998 & 9.8 & $(8.9-10.6)$ & 20.5 & $(19.5-21.6)$ & 15.2 & $(14.5-15.9)$ \\
\hline & 2002 & 9.2 & $(8.2-10.3)$ & 22.9 & $(21.6-24.2)$ & 16.2 & $(15.3-17.0)$ \\
\hline \multirow[t]{3}{*}{ Usual use of low fat, reduced fat or skim milk } & 1997 & 37.5 & $(36.0-38.9)$ & 53.8 & $(52.4-55.1)$ & 45.7 & $(44.7-46.7)$ \\
\hline & 1998 & 38.8 & $(37.3-40.3)$ & 52.4 & $(51.1-53.8)$ & 45.7 & $(44.7-46.7)$ \\
\hline & 2002 & 35.8 & $(34.0-37.6)$ & 50.7 & $(49.0-52.4)$ & 43.4 & $(42.1-44.6)$ \\
\hline Food insecurity last 12 months & 2002 & 5.2 & $(4.4-6.0)$ & 6.1 & $(5.3-6.9)$ & 5.7 & $(5.1-6.2)$ \\
\hline \multirow[t]{3}{*}{ Adequate physical activity } & 1997 & - & - & - & - & - & - \\
\hline & 1998 & 52.2 & $(50.7-53.7)$ & 43.1 & $(41.8-44.4)$ & 47.6 & $(46.6-48.6)$ \\
\hline & 2002 & 50.4 & $(48.4-52.3)$ & 42.9 & $(41.2-44.5)$ & 46.6 & $(45.3-47.8)$ \\
\hline \multirow[t]{3}{*}{ Current daily or occasional smoking } & 1997 & 27.2 & $(25.9-28.5)$ & 21.0 & $(20.0-22.0)$ & 24.0 & $(23.2-24.9)$ \\
\hline & 1998 & 26.2 & $(24.8-27.5)$ & 21.3 & $(20.2-22.4)$ & 23.7 & $(22.9-24.6)$ \\
\hline & 2002 & 23.9 & $(22.2-25.6)$ & 18.9 & $(17.6-20.2)$ & 21.4 & $(20.3-22.4)$ \\
\hline \multirow[t]{3}{*}{ Smoke-free households * } & 1997 & & & & & 69.8 & $(68.9-70.6)$ \\
\hline & 1998 & & & & & 73.2 & $(72.3-74.1)$ \\
\hline & 2002 & & & & & 81.0 & $(80.0-82.0)$ \\
\hline Over estimation of smokers in the community & 2002 & 79.8 & $(78.2-81.5)$ & 84.8 & $(83.5-86.2)$ & 82.3 & $(81.2-83.3)$ \\
\hline
\end{tabular}


In $2002,80.7$ per cent of the adult population rated their own health as 'excellent', 'very good', or 'good'. However this represents a decrease from the 1997 figure of 84.9 per cent.

In 2002, 10.6 per cent of people aged 16 years and over reported current asthma that was diagnosed by a doctor. The rate of current asthma has not altered since 1997.

In 2002, approximately six per cent of adults reported that a doctor had ever told them that they had diabetes.
The reported prevalence of diabetes has increased since 1997 from 4.7 per cent to 6.1 per cent.

Just under half of all people in 2002 reported being either overweight or obese. The proportion of people classified as overweight or obese has risen since 1997, from 42.2 per cent to 46.3 per cent.

One in eight people in 2002 reported either 'high' or 'very high' levels of psychological distress. Rates of 'high' and 'very high' psychological distress have risen since 1998, from 10.5 per cent to 12.2 per cent.

\section{TABLE 2}

TRENDS IN INDICATORS OF HEALTH STATUS BY SEX, NSW, 1997-2002

\begin{tabular}{|c|c|c|c|c|c|c|c|}
\hline \multirow{2}{*}{$\begin{array}{l}\text { Indicator } \\
\text { Excellent, very good, or good self-rated } \\
\text { health status }\end{array}$} & \multirow{2}{*}{$\begin{array}{c}\begin{array}{c}\text { Year } \\
\text { Collected }\end{array} \\
1997\end{array}$} & \multirow{2}{*}{$\begin{array}{c}\text { Males } \\
\% \\
84.9\end{array}$} & \multirow{2}{*}{$\begin{array}{c}(95 \% \mathrm{Cl}) \\
(83.9-85.8)\end{array}$} & \multicolumn{2}{|c|}{$\begin{array}{l}\text { Females }(95 \% \mathrm{Cl}) \\
\%\end{array}$} & \multicolumn{2}{|l|}{$\begin{array}{l}\text { Persons } \\
\%\end{array}$} \\
\hline & & & & 85.0 & $(84.1-85.9)$ & 84.9 & $(84.3-85.6)$ \\
\hline & 1998 & 84.9 & $(83.9-85.9)$ & 83.0 & $(82.1-83.9)$ & 83.9 & $(83.2-84.6)$ \\
\hline & 2002 & 81.8 & $(80.3-83.3)$ & 79.7 & $(78.5-81.0)$ & 80.7 & $(79.7-81.7)$ \\
\hline \multirow[t]{3}{*}{ Ever diagnosed with asthma } & 1997 & 14.9 & $(13.9-16.0)$ & 18.1 & $(17.1-19.2)$ & 16.6 & $(15.8-17.3)$ \\
\hline & 1998 & 15.4 & $(14.3-16.5)$ & 18.0 & $(17.0-19.0)$ & 16.7 & $(16.0-17.5)$ \\
\hline & 2002 & 18.3 & $(16.8-19.9)$ & 20.9 & (19.6-22.3) & 19.6 & $(18.6-20.7)$ \\
\hline \multirow[t]{3}{*}{ Current asthma } & 1997 & 8.7 & $(7.9-9.5)$ & 11.9 & $(11.0-12.8)$ & 10.3 & $(9.7-10.9)$ \\
\hline & 1998 & 8.9 & $(8.0-9.8)$ & 10.9 & $(10.1-11.7)$ & 9.9 & $(9.3-10.5)$ \\
\hline & 2002 & 9.1 & $(8.0-10.2)$ & 12.0 & $(11.0-13.0)$ & 10.6 & $(9.8-11.3)$ \\
\hline \multirow[t]{3}{*}{ Blood pressure measured within the last 2 years } & 1997 & 82.9 & $(81.7-84.0)$ & 91.7 & $(90.9-92.4)$ & 87.3 & $(86.7-88.0)$ \\
\hline & 1998 & 83.2 & $(82.0-84.4)$ & 91.8 & $(91.0-92.5)$ & 87.5 & $(86.8-88.2)$ \\
\hline & 2002 & 82.4 & $(80.8-84.0)$ & 90.8 & $(89.8-91.9)$ & 86.7 & $(85.8-87.7)$ \\
\hline \multirow[t]{3}{*}{ High blood pressure } & 1997 & 16.7 & $(15.6-17.7)$ & 16.1 & $(15.1-17.0)$ & 16.3 & $(15.7-17.0)$ \\
\hline & 1998 & 17.2 & $(16.1-18.4)$ & 17.1 & $(16.2-18.1)$ & 17.2 & $(16.5-17.9)$ \\
\hline & 2002 & 20.9 & $(19.4-22.4)$ & 19.0 & $(17.9-20.2)$ & 19.9 & $(19.0-20.9)$ \\
\hline \multirow[t]{3}{*}{ Cholesterol measured within last 2 years } & 1997 & 47.8 & $(46.4-49.3)$ & 46.6 & $(45.3-47.9)$ & 47.2 & $(46.2-48.2)$ \\
\hline & 1998 & 50.8 & $(49.3-52.4)$ & 47.5 & $(46.2-48.8)$ & 49.1 & $(48.1-50.2)$ \\
\hline & 2002 & 54.7 & $(52.6-56.7)$ & 52.4 & $(50.7-54.1)$ & 53.5 & $(52.2-54.8)$ \\
\hline \multirow[t]{3}{*}{ High cholesterol } & 1997 & 25.0 & $(23.4-26.5)$ & 23.6 & $(22.2-24.9)$ & 24.3 & $(23.2-25.3)$ \\
\hline & 1998 & 21.5 & $(20.0-23.0)$ & 21.4 & $(20.1-22.6)$ & 21.4 & $(20.5-22.4)$ \\
\hline & 2002 & 25.3 & $(23.4-27.3)$ & 24.4 & $(22.8-26.0)$ & 24.9 & $(23.6-26.1)$ \\
\hline Diagnosed chemical sensitivity & 2002 & 2.4 & $(1.9-3.0)$ & 3.4 & $(2.8-4.1)$ & 2.9 & $(2.5-3.4)$ \\
\hline \multirow[t]{3}{*}{ Diabetes or high blood sugar } & 1997 & 5.2 & $(4.6-5.8)$ & 4.3 & $(3.8-4.8)$ & 4.7 & $(4.3-5.1)$ \\
\hline & 1998 & 4.9 & $(4.2-5.5)$ & 4.0 & $(3.5-4.5)$ & 4.5 & $(4.0-4.9)$ \\
\hline & 2002 & 6.6 & $(5.8-7.4)$ & 5.7 & $(5.0-6.4)$ & 6.1 & $(5.6-6.7)$ \\
\hline Work related injury in last 12 months & 2002 & 17.9 & $(16.0-19.7)$ & 12.8 & $(11.3-14.3)$ & 15.6 & $(14.4-16.8)$ \\
\hline \multirow[t]{3}{*}{ High and very high psychological distress } & 1997 & 9.2 & $(8.4-10.0)$ & 12.9 & $(12.0-13.8)$ & 11.1 & $(10.5-11.7)$ \\
\hline & 1998 & 9.0 & $(8.1-9.9)$ & 12.0 & $(11.1-12.8)$ & 10.5 & $(9.9-11.1)$ \\
\hline & 2002 & 10.5 & $(9.3-11.6)$ & 14.0 & $(12.8-15.1)$ & 12.2 & $(11.4-13.1)$ \\
\hline \multirow[t]{3}{*}{ No natural teeth missing } & 1997 & - & - & - & - & - & - \\
\hline & 1998 & 36.3 & $(34.8-37.8)$ & 33.7 & $(32.4-35.0)$ & 35.0 & $(34.0-36.0)$ \\
\hline & 2002 & 37.9 & $(36.0-39.9)$ & 36.6 & $(34.9-38.2)$ & 37.2 & $(36.0-38.5)$ \\
\hline \multirow[t]{3}{*}{ Overweight and obesity } & 1997 & 49.7 & $(48.3-51.2)$ & 34.5 & $(33.3-35.8)$ & 42.2 & $(41.2-43.1)$ \\
\hline & 1998 & 50.3 & $(48.7-51.8)$ & 34.5 & $(33.2-35.7)$ & 42.5 & $(41.4-43.5)$ \\
\hline & 2002 & 53.9 & $(52.0-55.9)$ & 38.5 & $(36.9-40.1)$ & 46.3 & $(45.0-47.6)$ \\
\hline
\end{tabular}

Source: NSW Health Survey Program, Centre for Epidemiology and Research, NSW Department of Health. 
Between 1997 and 2002 there were increases in the proportion of people reporting they had ever been told they had high blood pressure (16.3 per cent to 19.9 per cent) and who had their cholesterol measured within two years (47.2 per cent to 53.5 per cent). Since 1997 there has been no change in the proportion of people reporting that they had their blood pressure last measured within two years (86.7 per cent).

\section{HEALTH SERVICES}

As part of the continuing commitment to monitoring satisfaction with health services in NSW, questions were asked about the use of and satisfaction with a range of services. These included difficulties getting health care when needed, admission to hospital, attendance at an emergency department, and use of community health centres and public dental services (Table 3).

In 2002, one in eight people reported experiencing difficulties getting health care when it was needed. Between 1997 and 2002 the proportion of people reporting difficulties getting health care when needed increased (10.0 per cent to 12.6 per cent).
Between 1997 and 2002 emergency department attendance in the previous 12 months ( 14.3 per cent) and hospital admission in the previous 12 months (13.9 per cent) remained unchanged. Of the people attending an emergency department in the previous 12 months, more than three-quarters rated the care received as 'excellent', 'very good', or 'good'. Of the people admitted to hospital in the last 12 months, over 90 per cent rated the care received as 'excellent', 'very good', or 'good'. Between 1997 and 2002 the proportion of people giving a positive rating of emergency department care (76.5 per cent) and hospital inpatient care (91.0 per cent) did not change between 1997 and 2002.

For the first time, information on attendance at and rating of public dental services and community health centres was collected and these will continue to be monitored in future surveys.

\section{SOCIAL CAPITAL}

The term 'social capital' refers to the institutions, relationships, and conventions that shape social networks, foster trust, and facilitate coordination and cooperation for mutual benefit. The New South Wales Adult Health

\section{TABLE 3}

TRENDS IN INDICATORS OF USE OF AND SATISFACTION WITH HEALTH SERVICES BY SEX, NSW, 1997-2002

\begin{tabular}{|c|c|c|c|c|c|c|c|}
\hline \multirow{4}{*}{$\begin{array}{l}\text { Indicator } \\
\text { Difficulties getting health care when needing it }\end{array}$} & \multirow{2}{*}{$\begin{array}{c}\begin{array}{c}\text { Year } \\
\text { Collected }\end{array} \\
1997\end{array}$} & \multirow{2}{*}{$\begin{array}{c}\begin{array}{c}\text { Males } \\
\%\end{array} \\
8.9\end{array}$} & \multirow{2}{*}{$\begin{array}{l}(95 \% \mathrm{Cl}) \\
(8.1-9.7)\end{array}$} & \multicolumn{2}{|c|}{$\underset{\%}{\text { Females }}(95 \% \mathrm{Cl})$} & \multicolumn{2}{|c|}{$\begin{array}{l}\text { Persons } \\
\%\end{array}$} \\
\hline & & & & 11.1 & $(10.4-11.9)$ & 10.0 & $(9.5-10.6)$ \\
\hline & 1998 & 8.6 & $(7.8-9.4)$ & 11.9 & $(11.1-12.6)$ & 10.3 & $(9.7-10.8)$ \\
\hline & 2002 & 10.9 & $(9.7-12.0)$ & 14.2 & $(13.1-15.3)$ & 12.6 & $(11.8-13.4)$ \\
\hline \multirow[t]{3}{*}{$\begin{array}{l}\text { Emergency department attendance in the } \\
\text { previous } 12 \text { months }\end{array}$} & 1997 & 15.7 & $(14.7-16.7)$ & 11.9 & $(11.1-12.7)$ & 13.8 & $(13.1-14.4)$ \\
\hline & 1998 & 13.9 & $(12.9-14.9)$ & 12.0 & $(11.2-12.8)$ & 12.9 & (12.3-13.6) \\
\hline & 2002 & 14.7 & $(13.4-16.0)$ & 13.8 & $(12.8-14.9)$ & 14.3 & $(13.4-15.1)$ \\
\hline \multirow[t]{3}{*}{$\begin{array}{l}\text { Emergency department care rated as } \\
\text { excellent, very good or good }\end{array}$} & 1997 & 80.5 & (77.7-83.4) & 79.9 & $(77.0-82.9)$ & 80.3 & $(78.2-82.3)$ \\
\hline & 1998 & 82.6 & $(79.5-85.6)$ & 78.6 & $(75.7-81.5)$ & 80.7 & $(78.6-82.8)$ \\
\hline & 2002 & 79.8 & (75.9-83.7) & 73.2 & $(69.3-77.0)$ & 76.5 & $(73.8-79.3)$ \\
\hline \multirow[t]{3}{*}{ Hospital admission in the previous 12 months } & 1997 & 11.3 & $(10.4-12.2)$ & 14.7 & $(13.8-15.5)$ & 13.0 & $(12.4-13.6)$ \\
\hline & 1998 & 11.5 & $(10.6-12.4)$ & 15.4 & $(14.5-16.3)$ & 13.5 & $(12.8-14.1)$ \\
\hline & 2002 & 11.3 & $(10.1-12.4)$ & 16.3 & $(15.1-17.6)$ & 13.9 & $(13.0-14.7)$ \\
\hline \multirow[t]{3}{*}{ Hospital care rated as excellent, very good or good } & 1997 & 90.3 & $(87.9-92.7)$ & 89.9 & $(87.9-91.9)$ & 90.1 & $(88.5-91.6)$ \\
\hline & 1998 & 92.5 & $(90.3-94.6)$ & 90.0 & $(88.1-91.9)$ & 91.0 & (89.6-92.5) \\
\hline & 2002 & 93.5 & $(90.7-96.2)$ & 89.3 & $(86.4-92.2)$ & 91.0 & (88.9-93.0) \\
\hline $\begin{array}{l}\text { Community health centre attendance in the } \\
\text { previous } 12 \text { months }\end{array}$ & 2002 & 4.8 & $(4.0-5.6)$ & 8.9 & $(8.0-9.9)$ & 6.9 & $(6.3-7.5)$ \\
\hline $\begin{array}{l}\text { Community health centre care rated as } \\
\text { excellent, very good or good }\end{array}$ & 2002 & 91.6 & (86.8-96.3) & 93.7 & $(91.0-96.4)$ & 92.9 & $(90.5-95.4)$ \\
\hline $\begin{array}{l}\text { Public dental service attendance in the } \\
\text { previous } 12 \text { months }\end{array}$ & 2002 & 3.9 & $(3.1-4.6)$ & 5.2 & $(4.4-5.9)$ & 4.5 & $(4.0-5.0)$ \\
\hline $\begin{array}{l}\text { Public dental service care rated as } \\
\text { excellent, very good or good }\end{array}$ & 2002 & 81.7 & $(74.4-89.1)$ & 80.7 & $(75.1-86.4)$ & 81.2 & $(76.7-85.6)$ \\
\hline
\end{tabular}

Source: NSW Health Survey Program, Centre for Epidemiology and Research, NSW Department of Health. 
TABLE 4

INDICATORS OF SOCIAL CAPITAL BY SEX, NSW, 2002

\begin{tabular}{|c|c|c|c|c|c|c|c|}
\hline Indicator & $\begin{array}{l}\text { Year } \\
\text { Collected }\end{array}$ & $\underset{\%}{\text { Males }}$ & $(95 \% \mathrm{Cl})$ & $\begin{array}{c}\text { Femal } \\
\%\end{array}$ & es $(95 \% \mathrm{Cl})$ & \multicolumn{2}{|c|}{$\begin{array}{l}\text { Persons }(95 \% \mathrm{Cl}) \\
\%\end{array}$} \\
\hline $\begin{array}{l}\text { Attended a community event at least once } \\
\text { in the last } 6 \text { months }\end{array}$ & 2002 & 52.9 & $(51.0-54.9)$ & 60.5 & $(58.9-62.1)$ & 56.8 & $(55.5-58.0)$ \\
\hline $\begin{array}{l}\text { Helped out any local group or organisation } \\
\text { at least once in the past } 3 \text { months }\end{array}$ & 2002 & 30.5 & $(28.7-32.2)$ & 35.7 & $(34.1-37.3)$ & 33.1 & $(32.0-34.3)$ \\
\hline $\begin{array}{l}\text { Active member of a local organisation, } \\
\text { church or club }\end{array}$ & 2002 & 45.5 & $(43.6-47.5)$ & 42.3 & $(40.7-43.9)$ & 43.9 & $(42.6-45.1)$ \\
\hline Most people can be trusted & 2002 & 69.0 & $(67.2-70.8)$ & 62.9 & $(61.3-64.6)$ & 65.9 & $(64.7-67.2)$ \\
\hline Feel safe walking down their street after dark & 2002 & 78.0 & $(76.4-79.5)$ & 55.8 & $(54.2-57.5)$ & 66.8 & $(65.6-67.9)$ \\
\hline Area has a reputation for being a safe place & 2002 & 75.2 & $(73.6-76.9)$ & 71.6 & $(70.1-73.1)$ & 73.4 & $(72.3-74.5)$ \\
\hline Visit neighbours & 2002 & 68.7 & $(66.9-70.5)$ & 63.2 & $(61.6-64.8)$ & 65.9 & $(64.7-67.1)$ \\
\hline $\begin{array}{l}\text { Able to ask for neighbourhood help to care } \\
\text { for a child }\end{array}$ & 2002 & 73.3 & $(71.5-75.1)$ & 68.0 & $(66.4-69.6)$ & 70.6 & $(69.4-71.8)$ \\
\hline $\begin{array}{l}\text { Run into friends and acquaintances when } \\
\text { shopping in local area }\end{array}$ & 2002 & 80.4 & $(78.8-82.0)$ & 83.7 & $(82.4-84.9)$ & 82.0 & $(81.1-83.0)$ \\
\hline Sad to leave neighbourhood & 2002 & 71.2 & $(69.4-73.0)$ & 75.7 & $(74.3-77.2)$ & 73.5 & $(72.4-74.7)$ \\
\hline
\end{tabular}

Survey 2002 included questions on social reciprocity and neighbourhood connection, feelings of trust and safety, and participation in the local community. This is the first time that questions on social capital have been included in an adult survey conducted by the NSW Health Survey Program (Table 4).

Seventy per cent of the population reported that they could ask someone in their neighbourhood for help with caring for a child, if they needed to; and nearly threequarters of the population said they would be sad if they had to leave their neighbourhood. Two-thirds of people reported feeling safe walking down their street after dark, and males were more likely to report feeling safe than females.

Overall, one-third of the population had helped out a local group or organisation, and more than half of the population had attended a local community event in the past six months.

\section{THE FUTURE}

The continued monitoring of indicators via the New South Wales Continuous Health Survey will provide information that will assist health professionals, health planners, and those involved in policy development to plan, implement, and evaluate health programs and initiatives within the community and within population target groups.

Printed copies of the New South Wales Adult Health Survey 2002 are available from the NSW Health Survey Program on (02) 94245707. Electronic copies can be downloaded in PDF format from the NSW Department of Health's website at www.health.nsw.gov.au/public-health/ phb/phb.html. 